\title{
ACTITUDES EUROPEAS ANTE EL ENVEJECIMIENTO Y LAS PERSONAS MAYORES
}

\author{
Alan Walker
}

Universidad de Sheffield

\section{INTRODUCCION}

La población de la Unión Europea está envejeciendo. En el año 2020, las personas mayores de 60 años sumarán una quinta parte de la población total de la UE, y las mayores de 65 superarán la cuarta parte. Hace solamente cincuenta años, estas últimas eran exactamente una de cada catorce personas de la población comunitaria. Sin embargo, esta revolución silenciosa de la estructura de la edad en Europa ha venido pasando bastante inadvertida para la población $\mathrm{y}$, hasta hace poco relativamente, también para los gestores de la política económica y social. Hasta 1993, por ejemplo, sabíamos muy poco acerca de las actitudes de la población ante las personas mayores en los diferentes países de la UE. Esta laguna del conocimiento se eliminó en buena medida con dos únicas encuestas de la opinión pública europea sobre el envejecimiento y la tercera edad, patrocinadas por la Comisión Europea para el Año europeo de la tercera edad y la solidaridad entre las generaciones. Se realizaron dos Eurobarómetros: en el primero se investigaban las actitudes de la población mayor de 15 años en cada uno de los doce países miembros de la UE, mientras que el segundo consistió en un estudio pormenorizado de las opiniones de las personas mayores. Estas dos encuestas relacionadas representaron el primer intento paneuropeo de proporcionar una imagen global de las actitudes ante el envejecimiento y la tercera edad. 
El objetivo de este artículo consiste en presentar un resumen de los principales resultados de estas encuestas. En la primera sección se consideran las actitudes de las personas mayores ante el envejecimiento; en la segunda, se examina el estado actual de la solidaridad entre las generaciones; en la tercera, se destacan los resultados clave para la política económica y social de los dos temas más importantes del envejecimiento de la sociedad -las pensiones y la atención prolongada- y, por último, se considera la política del envejecimiento. Inevitablemente, un breve artículo como éste sólo puede examinar superficialmente algunos de los resultados detallados, por lo que se recomienda a los lectores que buscan una descripción más completa que consulten Walker, 1993.

\section{Las encuestas}

Durante los últimos veintitrés años, la Comisión de las Comunidades Europeas, a través del DGX The Directorate-General for Information, Communication and Culture, ha organizado una encuesta bianual que incluye a cada uno de los países miembros (Reif e Inglehart, 1991). Las encuestas gemelas sobre las que se informa en este artículo eran el número 37 de las series y se realizaron entre el 20 de abril y el 18 de mayo de 1992. La primera fue una encuesta Eurobarómetro "normal» dirigida a la población de 15 y más años en cada uno de los países de la UE. La segunda fue una encuesta dirigida solamente a la población mayor de 60 años.

El Eurobarómetro normal es una encuesta de opinión realizada con una muestra de 1.000 personas, representativa de la población mayor de 15 años en cada uno de los doce países. En Luxemburgo, la muestra se limitó a 500 individuos, mientras que en Alemania se utilizó una de 1.000 individuos para la antigua RFA y otra de otros 1.000 para la antigua RDA, sumando juntas una muestra combinada de 2.000 personas. (En este informe se ha adoptado la fórmula de presentar los totales de las dos antiguas mitades de Alemania, además de la muestra combinada.) Para constituir el Reino Unido, a la muestra de Gran Bretaña se añadió una muestra adicional de 300 individuos para Irlanda del Norte. Sumando, por lo tanto, las muestras de los diferentes países, obtenemos una muestra total de unas 12.800 personas.

La encuesta complementaria para personas mayores consistió en muestras de 400 individuos de 60 y más años en todos los países miembros (200 en Luxemburgo, 800 en Alemania), obteniéndose, por lo tanto, en este caso, una muestra total de unos 5.000 individuos. En esta muestra, una de cada cuatro personas tenían entre 60 y 64 años de edad, y una proporción similar entre 65 y 69; una quinta parte tenía 70-74 años; el 16 por 100, 75-79, y el 14 por 100, 80 y más años. El 41 por 100 eran hombres y el 59 por 100 mujeres.

Se decidió que estas encuestas deberían proporcionar información básica sobre las actitudes ante las personas mayores y sobre algunos de los temas 
actuales en torno a la vejez, cuya regulación requiere medidas de política económica y social. En el caso de la encuesta a la población general, los principales temas suscitados comprendieron, entre otros, el nivel de vida y la idoneidad de las pensiones, la voluntad de la gente para financiarlas, el papel de las personas mayores en el mercado de trabajo, la necesidad y la oferta de ayuda o asistencia, y la medida en que las personas mayores deberían determinar sus propias necesidades de servicio. Los temas de política económica y social tratados en la encuesta complementaria para personas mayores comprendieron, entre otros, la edad de jubilación, el nivel de vida, las pensiones, la soledad y el aislamiento, los pagos en efectivo en lugar de exenciones, el apoyo social y las acciones políticas. Además, algunas preguntas estuvieron dirigidas específicamente al tema de la solidaridad intergeneracional para reflejar el objetivo del Año europeo de 1993.

De esta forma, la información recogida nos permite presentar por primera vez, en cada país miembro, una imagen de las actitudes de la población general ante el envejecimiento y las personas mayores, a la vez que perfilar unos rasgos generales de las condiciones y pensamientos actuales de los ciudadanos europeos de la tercera edad. También es posible informar sobre el estado actual de las relaciones entre las diferentes generaciones. Además de los datos esenciales sobre actitudes, cada Eurobarómetro proporciona otros tres tipos de información: datos demográficos, información sobre actitudes sociopsicológicas y sociopolíticas, así como respuestas a preguntas que muestran una tendencia en las opiniones sobre la integración europea y las percepciones de las instituciones europeas.

\section{EL CIUDADANO EUROPEO DE LA TERCERA EDAD}

En esta sección vamos a considerar las opiniones de las propias personas mayores. El principal objetivo es proporcionar unos rasgos generales de la experiencia subjetiva de ser mayor en la década de los noventa en cada uno de los países de la UE. El primer paso es considerar las opiniones de los mayores respecto al nombre que debiera dárseles como grupo.

\section{Un nombre colectivo}

Preguntamos a las personas mayores directamente cuál era el término de su preferencia para referirse a ellas como colectivo, y los resultados se encuentran en el cuadro 1. No hubo consenso en los países de la UE, estando dividida la opinión mayoritaria entre "personas mayores» y "ciudadanos de tercera edad». En cuatro países, la mayoría está a favor del término "personas mayores» y, en tres, del de "ciudadanos de tercera edad». Lo que ha quedado suficientemente 


\section{CUADRO 1}

La elección de un nombre (Sólo personas mayores)

\begin{tabular}{|c|c|c|c|c|c|c|c|c|c|c|c|c|c|c|c|}
\hline & $C E 12$ & Bélgica & $\begin{array}{l}\text { Dina- } \\
\text { marca }\end{array}$ & Francia & $\begin{array}{l}\text { Alem. } \\
\text { Occid. }\end{array}$ & $\begin{array}{l}\text { Alem. } \\
\text { Orien. }\end{array}$ & $\begin{array}{l}\text { Alem. } \\
\text { Occid. y } \\
\text { Orien. }\end{array}$ & Grecia & Irlanda & Italia & $\begin{array}{c}\text { Luxem- } \\
\text { burgo }\end{array}$ & Holanda & Portugal & España & $R U$ \\
\hline Ancianos & . 6,6 & 7,7 & 49,5 & 4,0 & 3,4 & 3,8 & 3,5 & 15,1 & 12,3 & 2,4 & 3,3 & 23,9 & 7,4 & 13,3 & 4,3 \\
\hline 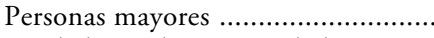 & 27,4 & 18,1 & 3,9 & 24,8 & 33,3 & 42,1 & 35,0 & 34,2 & 9,0 & 30,9 & 62,8 & 10,8 & 52,4 & 55,1 & 4,3 \\
\hline Ciudadanos de tercera edad ............. & . 30,9 & 31,5 & 18,5 & 21,5 & 47,1 & 35,1 & 44,7 & 14,7 & 41,9 & 21,1 & 15,7 & 13,0 & 21,7 & 14,5 & 45,2 \\
\hline 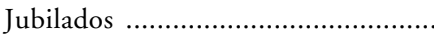 & . 15,0 & 32,6 & 24,5 & 26,5 & 12,2 & 17,1 & 13,1 & 17,6 & 14,4 & 9,1 & 18,2 & 5,5 & 13,3 & 7,8 & 14,7 \\
\hline Viejos/personas de edad avanzada ... & . 10,6 & 0,0 & 0,0 & 21,1 & 1,0 & 0,3 & 0,9 & 4,7 & 4,5 & 36,5 & 0,0 & 18,7 & 0,0 & 0,3 & 0,8 \\
\hline 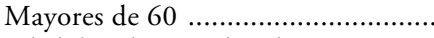 & .. 1,1 & 4,7 & 0,0 & 0,0 & 0,0 & 0,0 & 0,0 & 0,0 & 0,0 & 0,0 & 0,0 & 24,6 & 0,0 & 0,0 & 0,0 \\
\hline Edad dorada/años dorados/viejecitos . & . 0,2 & 0,0 & 0,0 & 0,7 & 0,0 & 0,0 & 0,0 & 0,0 & 5,1 & 0,0 & 0,0 & 0,0 & 0,0 & 0,0 & 0,0 \\
\hline 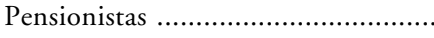 & .. 1,1 & 0,0 & 0,0 & 0,0 & 0,0 & 0,0 & 0,0 & 0,0 & 9,3 & 0,0 & 0,0 & 0,0 & 0,0 & 0,2 & 5,8 \\
\hline 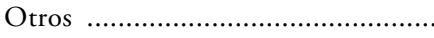 & .. 5,5 & 0,0 & 3,4 & 1,0 & 0,7 & 0,7 & 0,7 & 11,8 & 0,0 & 0,0 & 0,0 & 1,6 & 1,9 & 8,0 & 22,9 \\
\hline 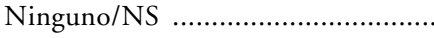 & .. 1,6 & 5,4 & 0,2 & 0,4 & 2,3 & 0,9 & 2,1 & 1,9 & 3,5 & 0,0 & 0,0 & 1,9 & 3,3 & 0,8 & 2,8 \\
\hline
\end{tabular}

La pregunta exacta era "De los siguientes términos para describir a las personas mayores de 60 años, ¿cuál es el que usted prefiere utilizar?» (Los entrevistados podían añadir otro de su elección.) 
claro, no obstante, es que, a excepción de Dinamarca y, parcialmente, de Holanda, el término «ancianos», que es el más frecuentemente utilizado por los gestores de la política económica y social, los medios de comunicación y los gerontólogos sociales, se rechaza con firmeza*.

En algunos países parece existir una reacción en contra de las connotaciones de la «vejez». Este hecho quedó al descubierto en la encuesta de actitudes ante al envejecimiento de 1991 en el Reino Unido, y la baja proporción de ciudadanos de tercera edad de este país que votaron por el término "personas mayores», "pensionistas" y "ancianos» confirma esos resultados de encuesta. Sin embargo, solamente Dinamarca y, en menor medida, Irlanda y Holanda comparten esta reacción y, si se les excluye junto con el Reino Unido, el término favorito, con gran diferencia, es el de «personas mayores». En contraste, los italianos parecen adherirse por completo al concepto de vejez: dos de cada tres personas prefieren "personas mayores» o «viejos»/«personas de edad avanzada».

Estos resultados son importantes porque los distintivos o etiquetas poseen significados simbólicos y, especialmente en este caso, nos dicen muchas cosas sobre el papel social y el estatus de los etiquetados. Una conclusión que podría obtenerse de estos resultados es que los profesionales responsables de poner un nombre a este grupo creciente de ciudadanos europeos no han estado suficientemente atentos hasta la fecha a lo que los propios interesados quieren que se les llame.

\section{¿Respeto hacia nuestros mayores?}

¿Se trata con más respeto a las personas cuando llegan a la vejez o acaso ocurre lo contrario? La respuesta para la UE en su totalidad es que tres de cada diez personas, aproximadamente, dicen que se las trata con más respeto y una proporción ligeramente inferior dice que con menos, lo que nos deja con una mayoría que (espontáneamente) responde no haber detectado ninguna diferencia de actitud hacia ellos. Resulta interesante observar lo que aparece como una cierta asociación entre la edad y el tratamiento que las personas mayores perciben que reciben de los demás: el 26 por 100 de las personas entre 60 y 64 años afirma que se las trata con más respeto, proporción que alcanza el 35 por 100 entre los mayores de 75 años. Las variaciones entre países se muestran en el cuadro 2.

Para concretar más precisamente si las personas mayores han experimentado, o no, actitudes paternalistas, denigrantes, despectivas o discriminatorias por su edad en algún establecimiento o institución, les hemos preguntado si alguna vez han recibido ese tratamiento por parte de un amplio abanico de

* Nota de la traductora: El término en inglés es elderly. 


\section{CUADRO 2}

Proporción de personas mayores que dicen que se las trata con más, o menos, respeto al ir envejeciendo

(En porcentajes)

\begin{tabular}{|c|c|c|}
\hline & Más respeto & Menos respeto \\
\hline 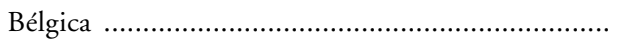 & 19 & 33 \\
\hline Dinamarca & 19 & 15 \\
\hline 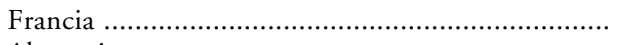 & 34 & 26 \\
\hline 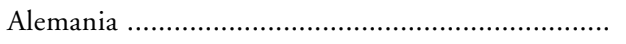 & 24 & 31 \\
\hline 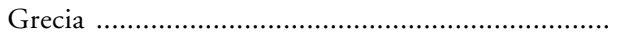 & 43 & 30 \\
\hline Irlanda & 44 & 21 \\
\hline 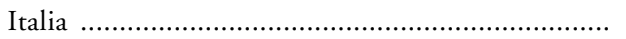 & 33 & 26 \\
\hline 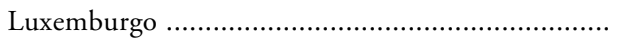 & 29 & 21 \\
\hline 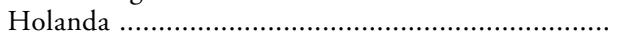 & 20 & 28 \\
\hline 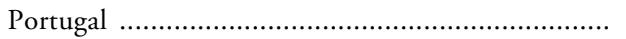 & 34 & 13 \\
\hline 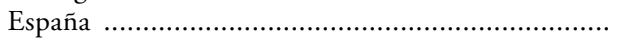 & 33 & 28 \\
\hline 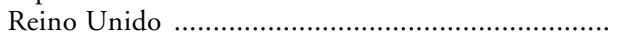 & 34 & 25 \\
\hline
\end{tabular}

diferentes organismos públicos y comerciales, de los profesionales, los medios de comunicación y, por último, sus propias familias. El hecho más destacable que surge de esta serie de preguntas es la escasa proporción de personas mayores que afirman haber sido tratadas alguna vez como ciudadanos de segunda categoría (un 19 por 100 por funcionarios locales y otro 18 por 100 por empleados de la Seguridad Social).

\section{¿Es mejor envejecer?}

¿Cómo responden las personas mayores al proceso de envejecimiento? Para poder calibrar este tema les preguntamos que si el hecho de envejecer les ha resultado de alguna forma tonificante o estimulante. Esta pregunta tenía, obviamente, la intención de eliminar las connotaciones negativas del envejecimiento y presentar su posible lado positivo. Los resultados, como muestra el cuadro 3, fueron sorprendentes. La media de las cuatro categorías de respuesta se sitúa, más o menos, en el punto medio entre el acuerdo y el desacuerdo, como si los ciudadanos mayores de la UE estuvieran expresando una cierta ambigüedad en la experiencia. Quizá se trate, en su opinión, como el resto de la vida, de buenos y malos aspectos.

Si observamos las variaciones entre países, la diferencia entre Dinamarca y 


\section{CUADRO 3}

La vejez me ha resultado tonificante

(Sólo personas mayores)

心

\begin{tabular}{|c|c|c|c|c|c|c|c|c|c|c|c|c|c|c|c|}
\hline & CE12 & Bélgica & $\begin{array}{l}\text { Dina- } \\
\text { marca }\end{array}$ & Francia & $\begin{array}{l}\text { Alem. } \\
\text { Occid. }\end{array}$ & $\begin{array}{l}\text { Alem. } \\
\text { Orien. }\end{array}$ & $\begin{array}{l}\text { Alem. } \\
\text { Occid. y } \\
\text { Orien. }\end{array}$ & Grecia & Irlanda & Italia & $\begin{array}{c}\text { Luxem- } \\
\text { burgo }\end{array}$ & Holanda & Portugal & España & $R U$ \\
\hline Muy de acuerdo & . 14,8 & 8,7 & 23,8 & 11,7 & 8,9 & 6,8 & 8,5 & 6,5 & 14,6 & 15,3 & 19,8 & 11,0 & 9,5 & 27,4 & 21,9 \\
\hline Algo de acuerdo & .. 30,0 & 26,9 & 38,1 & 32,4 & 27,1 & 17,0 & 25,1 & 12,7 & 29,3 & 38,4 & 26,2 & 13,8 & 25,7 & 32,9 & 32,2 \\
\hline Algo en desacuerdo & .. 31,0 & 43,0 & 20,4 & 32,2 & 37,1 & 40,9 & 37,8 & 30,3 & 29,0 & 28,1 & 29,9 & 36,1 & 25,1 & 19,9 & 27,9 \\
\hline Muy en desacuerdo & .. 19,6 & 16,2 & 12,2 & 19,1 & 22,7 & 30,8 & 24,3 & 41,1 & 22,6 & 12,5 & 11,7 & 32,3 & 34,8 & 14,7 & 15,5 \\
\hline No sabe & .. 4,6 & 5,2 & 5,5 & 4,7 & 4,2 & 4,4 & 4,3 & 9,5 & 4,5 & 5,8 & 12,4 & 6,8 & 5,0 & 5,0 & 2,3 \\
\hline
\end{tabular}


Grecia en las proporciones que están muy de acuerdo con la frase es, sin duda, atribuible parcialmente a las mejores condiciones objetivas de las personas mayores danesas en comparación con sus homólogas griegas (véase Walker, Guillemard y Alber, 1993). Pero el hecho de que en España esa proporción sea incluso más alta sugiere que las condiciones materiales sólo ofrecen una explicación parcial del tema. En cuanto a la edad, las diferencias son relativamente pequeñas: el 51 por 100 estaba de acuerdo con que el envejecimiento les ha resultado tonificante, comparado con el 41 por 100 de las personas mayores de 75 años. No es de extrañar que las personas mayores con discapacidades mostraran menor predisposición a responder positivamente a esta pregunta: el 39 por 100 de este grupo, frente al 49 por 100 de los no discapacitados.

\section{Intimidad a distancia}

En todos los países comunitarios se observa una tendencia al aumento del número de personas mayores que viven solas, si bien está más pronunciada en el Norte que en el Sur. La segregación residencial, por lo tanto, ha suscitado el temor a que las personas mayores estén siendo abandonadas por sus familiares. Datos de investigaciones, sin embargo, no confirman este hecho en absoluto, ya que, en efecto, las personas mayores mantienen unos lazos muy estrechos con sus familiares, y viceversa, una posición que ha quedado perfectamente descrita como de «intimidad a distancia» (Rosemayer y Kockels, 1996). Debido a que éste continúa siendo un tema de actualidad y a que resulta difícil obtener información en algunos países de la UE, decidimos preguntar directamente a las personas mayores sobre sus contactos con la familia. Los resultados aparecen en el cuadro 4 y confirman los de otras investigaciones en diferentes países, pudiéndose observar contactos personales frecuentes entre las personas mayores y sus familiares. Como promedio, cerca de cuatro de cada cinco personas ven a un miembro de su familia al menos una vez por semana.

Se formuló una pregunta similar acerca de los amigos y, de nuevo, los resultados muestran la existencia de un fuerte contacto social: como promedio, casi tres de cada cuatro personas ven a un amigo al menos una vez por semana. Pero existían grandes diferencias entre países en este punto: en Alemania y Holanda no llegaban a una de cada diez, mientras que en Portugal y España eran seis de cada diez.

A pesar del fuerte contacto social, los sentimientos de soledad están presentes en una minoría significativa de personas mayores. En conjunto, aproximadamente una de cada ocho personas dijo que se sentía sola frecuentemente, y una de cada tres, ocasionalmente. Pero existen grandes variaciones entre países (cuadro 5). 


\section{CUADRO 4}

Contactos con la familia

(Sólo personas mayores)

\begin{tabular}{|c|c|c|c|c|c|c|c|c|c|c|c|c|c|c|c|}
\hline & CE12 & Bélgica & $\begin{array}{l}\text { Dina- } \\
\text { marca }\end{array}$ & Francia & $\begin{array}{l}\text { Alem. } \\
\text { Occid. }\end{array}$ & $\begin{array}{l}\text { Alem. } \\
\text { Orien. }\end{array}$ & $\begin{array}{l}\text { Alem. } \\
\text { Occid. y } \\
\text { Orien. }\end{array}$ & Grecia & Irlanda & Italia & $\begin{array}{c}\text { Luxem- } \\
\text { burgo }\end{array}$ & Holanda & Portugal & España & $R U$ \\
\hline Todos los días & 44,4 & 35,8 & 13,8 & 34,2 & 48,7 & 37,7 & 46,5 & 64,8 & 50,1 & 70,7 & 38,0 & 19,2 & 59,8 & 60,7 & 21,9 \\
\hline Dos o más veces por semana ........... & 18,3 & 22,0 & 26,0 & 16,2 & 15,2 & 17,6 & 15,6 & 9,7 & 19,3 & 14,4 & 21,1 & 26,6 & 9,6 & 15,5 & 28,3 \\
\hline Una vez por semana $\ldots \ldots \ldots \ldots \ldots \ldots \ldots \ldots \ldots \ldots \ldots \ldots \ldots$ & 15,5 & 23,5 & 25,4 & 25,1 & 13,0 & 17,4 & 13,8 & 5,8 & 14,1 & 7,8 & 19,4 & 25,4 & 8,7 & 7,4 & 19,0 \\
\hline Una vez cada quince días ................ & 6,1 & 4,7 & 16,9 & 5,8 & 9,9 & 6,4 & 9,2 & 4,2 & 2,5 & 1,2 & 9,5 & 10,8 & 3,8 & 3,8 & 6,8 \\
\hline Una vez al mes ............................... & . 4,6 & 3,5 & 7,8 & 6,8 & 4,4 & 9,6 & 5,4 & 2,0 & 3,8 & 0,8 & 3,5 & 8,9 & 4,2 & 2,9 & 5,6 \\
\hline Menos frecuentemente .................... & . 7,6 & 6,0 & 8,2 & 9,0 & 4,9 & 7,9 & 5,5 & 7,3 & 6,8 & 1,6 & 6,5 & 5,8 & 10,1 & 8,3 & 14,5 \\
\hline Nunca/no tengo familia ni amigos ... & 3,4 & 4,4 & 1,8 & 3,0 & 4,0 & 3,6 & 3,9 & 6,3 & 3,4 & 3,4 & 3,1 & 3,2 & 3,8 & 1,4 & 4,0 \\
\hline
\end{tabular}




\section{CUADRO 5}

Proporción de personas mayores

que se sienten solas frecuentemente

\begin{tabular}{ll}
\hline \multicolumn{1}{c}{$\%$} & \multicolumn{1}{c}{ País de la UE } \\
\hline$<5$ & Dinamarca \\
$5-9$ & Alemania, Holanda, Reino Unido \\
$10-14$ & Bélgica, Francia, Irlanda, Luxemburgo, España \\
$15-19$ & Italia \\
20 o más & Portugal, Grecia (36\%) \\
\hline
\end{tabular}

\section{RELACIONES INTERGENERACIONALES}

En esta sección examinamos los resultados de la encuesta desde la perspectiva de uno de los temas más importantes para el futuro de la sociedad europea: las relaciones intergeneracionales. La literatura está salpicada de referencias al mismo — los hijos de Job, Edipo, el rey Lear y sus hijas-, por lo que sabemos que se trata de un tema muy antiguo. Pero ¿cuál es el estado actual de las relaciones entre las generaciones? ¿Qué es lo que piensan las personas mayores de la gente joven? ¿Están las personas mayores a favor de la segregación por la edad? Y, algo fundamental para la política económica y social, ¿continuará la población en su conjunto pagando las pensiones de las generaciones de personas mayores?

Es un tópico conocido la falta de respeto hacia las personas mayores por parte de los jóvenes, razón por lo que preguntamos a los ciudadanos mayores europeos si estaban o no de acuerdo con la frase de que "los jóvenes por lo general ayudan a las personas mayores». La formulación de la pregunta fue intencionadamente positiva para evitar la promoción de un estereotipo popular negativo. Los resultados indican que la gente mayor está, por lo general, bien dispuesta hacia los jóvenes. Los más positivos en sus opiniones sobre los jóvenes son los daneses e irlandeses, en contraste con los belgas e italianos, que son los menos. Las personas en su cuarta edad mostraban mayor predisposición a estar muy de acuerdo con la frase de que los jóvenes ayudan a las personas mayores que las de la tercera edad.

En 1990 se habían realizado unas encuestas Eurobarómetro a jóvenes de entre 15 y 24 años y esto nos permitió comparar distintas generaciones en la respuesta a una pregunta concreta (Comisión CE, 1990). La pregunta elegida se refiere a una lista de cualidades que los padres pueden intentar fomentar en sus hijos, incluidas la independencia, tolerancia, lealtad, etc. A los entrevistados se les pidió que eligieran tres categorías de respuesta de un total de once (o que las rechazaran). Los resultados pueden observarse en el cuadro 6 . 


\section{CUADRO 6}

Cualidades que los padres desean fomentar en sus hijos (En porcentajes)

Grupos de edad

\begin{tabular}{|c|c|c|}
\hline \multirow{3}{*}{ Sentido de la responsabilidad ................................... } & \\
\hline & $\begin{array}{l}15-24 \\
(1990)\end{array}$ & $\begin{array}{c}60 \text { y más } \\
\text { (1992) }\end{array}$ \\
\hline & 55 & 57 \\
\hline 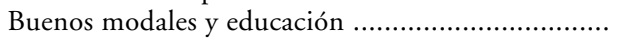 & 48 & 54 \\
\hline 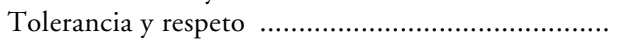 & 43 & 45 \\
\hline 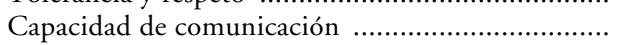 & 35 & 11 \\
\hline Independencia & 27 & 11 \\
\hline Lealtad & 20 & 21 \\
\hline 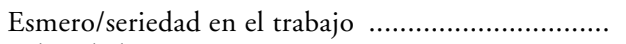 & 19 & 28 \\
\hline 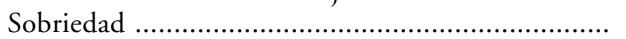 & 13 & 31 \\
\hline Imaginación & 12 & 3 \\
\hline 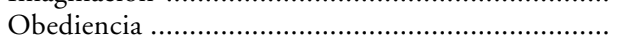 & 11 & 17 \\
\hline 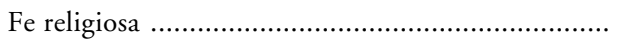 & 6 & 16 \\
\hline 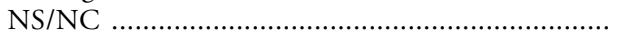 & 2 & 1 \\
\hline
\end{tabular}

La similitud entre jóvenes y mayores a la hora de seleccionar las primeras tres cualidades es extraordinaria, aunque a partir de ahí parece acabarse el consenso, excepto en un solo caso: la lealtad. Podría decirse que dos categorías, la capacidad de comunicación y la independencia, son más valoradas por los jóvenes, mientras que la seriedad en el trabajo, la sobriedad, la obediencia y la fe religiosa parecen claras favoritas de las personas mayores. No obstante, la impresión principal es que reina el consenso en las tres cualidades consideradas como las más importantes.

También se intentó que el cuestionario de la encuesta a personas mayores fuese comparable al estudio de 1990 de actitudes de los jóvenes ante los temas más importantes de la actualidad, pero esto no fue posible y tuvimos que contentarnos con una formulación diferente de la pregunta. A pesar de ello, resulta posible obtener una comparación aproximada, orientativa, entre algunos de los temas defendidos por los jóvenes y por los mayores.

Como promedio, casi la mitad de las personas mayores estaban interesadas en el medio ambiente (el 69 por 100 en Luxemburgo y el 30 por 100 en España). En la encuesta de 1990, el 56 por 100 de los jóvenes de 15 a 24 años consideraba que ésta era una de sus "grandes causas». Algo más de dos de cada cinco personas mayores están interesadas en problemas sociales importantes, como los derechos humanos y la pobreza (54 por 100 en Grecia, 26 por 100 en Bélgica), mientras que algo menos de dos de cada cinco jóvenes dijeron que la pobreza era una de sus grandes causas y casi la mitad se decantaba por los 
derechos humanos. La proporción de jóvenes interesados en temas del Tercer Mundo es el doble de la de personas mayores (el 29 frente al 14 por 100). La paz mundial era la causa dominante en la encuesta de jóvenes de 1990 (el 60 por 100), mientras que entre los mayores, por el contrario, el interés decaía considerablemente: sólo algo más de uno de cada diez (el 36 por 100 en Grecia, el 4 por 100 en España). Por último, la proporción de personas mayores interesadas en la religión era más de cuatro veces mayor que la sugerida para los jóvenes en la encuesta de 1990 (el 32 por 100 y el 7 por 100, respectivamente).

\section{¿Segregación por la edad?}

Para poder evaluar el estado actual de las relaciones intergeneracionales es esencial conocer el grado de contacto que existe entre ellas. Con este objetivo se formuló la siguiente pregunta directa: «¡cuánto contacto mantiene usted con gente joven, digamos que, menor de veinticinco años, incluyendo los miembros de su propia familia?». Los resultados revelaban una fuerte interacción entre jóvenes y viejos. Como era de esperar, el contacto disminuye con la edad: el 46 por 100 de las personas de 60-64 años dicen que tienen mucho contacto, proporción que se reduce al 27 por 100 a partir de los 75 años. Un 6 por 100 en el primer grupo y un 14 en el segundo afirman carecer de todo contacto. En España, las personas mayores de 60 años mostraban una predisposición menor que en ningún otro país a decir que mantienen mucho contacto con gente joven (el 26 por 100, comparado con una media de la UE del 36 por 100 y un máximo del 46 por 100 en Irlanda).

A continuación, se hacía la siguiente pregunta complementaria: «¿le gustaría tener más contacto con gente joven?». Los resultados sugieren ambivalencia: un 45 por 100 desea más y otro 25 por 100 no lo desea (con un 10 por 100 de "no sabe»). Sin embargo, cuando estos porcentajes se desagregan por países, surge una imagen clara: más de tres de cada cinco personas mayores en Grecia, Italia y Portugal dijeron que deseaban más contacto con gente joven, mientras que proporciones similares en Dinamarca, Holanda y el Reino Unido dijeron, por el contrario, que no lo deseaban.

Se formularon algunas preguntas para investigar el tema de la segregación por la edad desde la perspectiva de las personas mayores. Una de ellas era si creen que las personas mayores prefieren relacionarse con gente de su misma edad. Una mayoría estaba de acuerdo en que sí: el 24 por 100 muy de acuerdo y el 35 por 100 algo de acuerdo, mientras que solamente el 11 por 100 estaba muy en desacuerdo. En Grecia y Portugal se dio la mayor concentración de acuerdo con esta frase (el 44 por 100 estaba muy de acuerdo), seguidos de España (36 por 100), Irlanda y Luxemburgo (31 por 100). Los mayores desacuerdos se dieron en Dinamarca (el 26 por 100 estaba muy en desacuerdo), el Reino Unido (18 por 100) y Holanda (16 por 100). 
Centrándonos en el tema desde una perspectiva política y teniendo en cuenta el surgimiento, en Estados Unidos, de un tipo de acción política basada en los «intereses de la edad», nos preguntamos si las personas mayores estarían dispuestas a formar parte de un partido político formado especialmente para promover sus intereses. La mayoría se oponía a la idea, pero la minoría —una media del 22 por 100 en los países de la UE- parece sorprendentemente grande. En el cuadro 7 se puede observar una desagregación por países.

\section{CUADRO 7}

Proporción de personas mayores que dijeron que entrarian a formar parte de un partido politico cuyo objetivo fuera defender sus intereses

(En porcentajes)

\begin{tabular}{|c|c|c|c|}
\hline 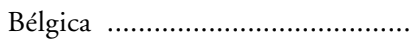 & 16 & 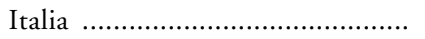 & 34 \\
\hline 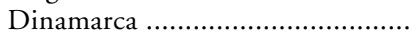 & 20 & 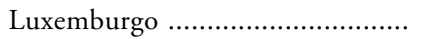 & 21 \\
\hline Francia .............. & 22 & Holanda .. & 21 \\
\hline 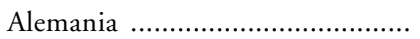 & 14 & 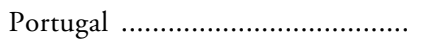 & 42 \\
\hline 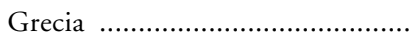 & 33 & 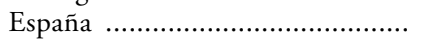 & 18 \\
\hline Irlanda & 16 & 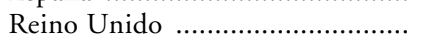 & 20 \\
\hline
\end{tabular}

Los resultados de esta sección de la encuesta a personas mayores sugieren que la interacción entre jóvenes y viejos es relativamente frecuente y que, en opinión de las personas mayores, esta interacción es suficiente en la mayoría de los países. Parece, sin embargo, que los ciudadanos de la tercera edad piensan que su propio colectivo prefiere relacionarse con personas de su misma edad. Además, parece existir una minoría importante en una mayoría de Estados miembros que apoyaría el surgimiento de partidos políticos relacionados con la edad.

Este tema también se investigó en la encuesta realizada a la población general y, de nuevo, se optó por un planteamiento positivo: se preguntaba si se estaba de acuerdo o en desacuerdo con que «la gente joven admira y respeta a las personas mayores». Una mayoría del 63 por 100 estaba en desacuerdo (dos terceras partes algo en desacuerdo y una tercera parte muy en desacuerdo). Las principales desviaciones de esta opinión se produjeron en Irlanda y Portugal, donde más de una quinta parte estaba muy de acuerdo con la frase y más de la mitad algo de acuerdo o muy de acuerdo.

Por lo tanto, en opinión de la población general en la mayoría de los Estados miembros, los jóvenes no admiran ni respetan a los mayores. Cuando desagregamos las respuestas en términos de la edad, nos encontramos con que los más jóvenes (15-24 años) se inclinaban algo más por una respuesta afirmativa que las personas mayores de 55 años: el 38 por 100 de los primeros estaba de acuerdo en que los jóvenes admiran y respetan a los mayores, frente al 31 por 
100 de las personas mayores de 55 años. Una mayoría de la población general europea también mantiene que las personas mayores son demasiado rígidas en sus hábitos e ideas: el 69 por 100 (una tercera parte estaba muy de acuerdo y dos terceras partes algo de acuerdo). De nuevo, los niveles mayores de desacuerdo se encontraban en Dinamarca e Irlanda, donde el 17 y el 13 por 100, respectivamente, estaban en desacuerdo, en contraste con una media del 7 por 100 para la UE en su conjunto.

La idea de que «a las personas mayores no les gusta escuchar las opiniones de los jóvenes» dividió las posibles respuestas casi exactamente por la mitad en la media de la UE. Los países que mostraban mayor acuerdo fueron Portugal, Luxemburgo y España, y los que estaban más en desacuerdo, Dinamarca, Irlanda y Holanda. En la encuesta a la población general también se preguntó si «las personas mayores y los jóvenes deberían relacionarse más frecuentemente». La respuesta fue unánime: sí deberían, en opinión de nueve de cada diez entrevistados, divididos por igual entre los que se mostraban algo de acuerdo y muy de acuerdo. Los únicos países que se desviaban algo de esta tendencia fueron Bélgica y el Reino Unido, pero, incluso en estos, existían grandes mayorías a favor de las relaciones intergeneracionales.

\section{La protección de los mayores}

Finalmente, en esta sección nos enfrentamos al tema macroeconómico fundamental de las relaciones entre las generaciones: la financiación de las pensiones. En todos los Estados miembros de la UE se ha manifestado recientemente la preocupación por las implicaciones del envejecimiento de la población para la política económica y social, preocupación que se suele centrar en un punto concreto: la creciente "relación de dependencia» entre las personas mayores y las que están en edad laboral. En otro lugar se han expuesto las deficiencias de estas crudas herramientas demográficas; no obstante, estas relaciones de dependencia constituyen un motivo serio de preocupación para algunos gestores de la política económica y comentaristas independientes. Por ejemplo, algunos expertos han señalado la capacidad de conflicto latente entre los trabajadores y los pensionistas, si no se elimina la "carga" de tener que financiar las pensiones (Johnson, Conrad Thompson, 1989).

Es esencial, si se va a producir un debate serio sobre el tema, saber lo que piensa la gente sobre el pago de impuestos para financiar las pensiones. De su voluntad para continuar haciéndolo depende el contrato social que sirve de sostén a las pensiones y los sistemas de protección social de la UE (y otros lugares). Abordamos este tema en el Eurobarómetro normal (realizado entre la población de 15 y más años), preguntando en qué medida se estaba de acuerdo o en desacuerdo con la frase de que las personas con empleo tienen el deber de asegurar, mediante su contribución en impuestos, un nivel de vida decente para las personas mayores.

Los resultados del cuadro 8 muestran un grado muy elevado de consenso y 


\section{CUADRO 8}

Las personas con empleo tienen el deber de asegurar, mediante sus contribuciones en impuestos, un nivel de vida digno para los mayores

\begin{tabular}{|c|c|c|c|c|c|c|c|c|c|c|c|c|c|c|c|}
\hline & CE12 & Bélgica & $\begin{array}{l}\text { Dina- } \\
\text { marca }\end{array}$ & Francia & $\begin{array}{l}\text { Alem. } \\
\text { Occid. }\end{array}$ & $\begin{array}{l}\text { Alem. } \\
\text { Orien. }\end{array}$ & $\begin{array}{l}\text { Alem. } \\
\text { Occid. y } \\
\text { Orien. }\end{array}$ & Grecia & Irlanda & Italia & $\begin{array}{c}\text { Luxem- } \\
\text { burgo }\end{array}$ & Holanda & Portugal & España & $R U$ \\
\hline 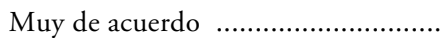 & 37,0 & 32,5 & 60,1 & 25,9 & 28,5 & 37,6 & 30,4 & 39,4 & 40,7 & 38,4 & 34,2 & 42,4 & 41,2 & 45,7 & 45,9 \\
\hline 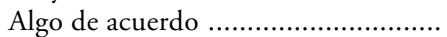 & 42,8 & 42,7 & 29,8 & 51,2 & 49,7 & 43,7 & 48,4 & 35,0 & 40,9 & 40,1 & 44,8 & 38,2 & 32,3 & 38,1 & 37,2 \\
\hline 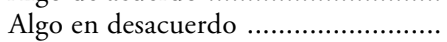 & . 9,0 & 13,8 & 6,3 & 13,0 & 12,4 & 7,7 & 11,4 & 8,3 & 5,8 & 6,9 & 10,6 & 9,8 & 10,0 & 4,4 & 6,2 \\
\hline Muy en desacuerdo .......................... & 8,6 & 4,1 & 2,0 & 4,6 & 3,7 & 3,2 & 3,6 & 4,2 & 1,7 & 2,7 & 3,4 & 4,0 & 7,8 & 2,8 & 3,1 \\
\hline No sabe & . 7,6 & 6,9 & 1,8 & 5,3 & 5,8 & 7,8 & 6,2 & 13,1 & 10,9 & 11,1 & 7,0 & 5,6 & 8,7 & 9,1 & 7,5 \\
\hline
\end{tabular}

No sabe

.


sugieren que el contrato social está en buena forma. Si hubiera de inquietarnos algún indicio en sentido contrario, tendría que ver con la tendencia observada entre las personas de 15 a 24 años y de 25 a 34 a no estar tan de acuerdo con la frase como otros grupos de edad. Por ejemplo, el 28 por 100 de los jóvenes de 15 a 24 años estaba muy de acuerdo con el contrato social, comparado con el 40 y 41 por 100 de las personas entre 55 y 64 años y las mayores de 65, respectivamente. Sin embargo, los jóvenes mostraban una mayor predisposición a estar algo de acuerdo, por lo que el consenso global se mantenía.

\section{TEMAS CLAVE DE POLITICA ECONOMICA Y SOCIAL}

Esta sección proporciona un breve resumen de algunos de los resultados de encuesta que son clave para la política económica y social, debido al envejecimiento de las sociedades europeas.

\section{Ingresos}

En estas encuestas nuestro interés se centraba en las opiniones subjetivas de las personas mayores y de la población general, ya que los datos objetivos sobre los ingresos de los pensionistas en los diferentes países de la UE pueden encontrarse en otras fuentes (Walker, Guillemard y Alber, 1993).

A los jubilados de la encuesta de la población general se les preguntó si las pensiones que recibían (públicas y privadas) eran suficientes. Los resultados dividieron a la Comunidad en tres grupos: los países con una mayoría de personas mayores que consideran que sus pensiones son adecuadas (Dinamarca, Alemania, Luxemburgo y Holanda); aquellos otros donde la opinión está dividida entre los que las consideran suficientes e insuficientes (Bélgica, Francia, Irlanda, Italia, España y el Reino Unido); y, por último, aquellos en los que grandes mayorías afirman que las pensiones son insuficientes (Grecia y Portugal). Sin olvidar que estos resultados no reflejan datos objetivos, conviene tener en cuenta que, desde la perspectiva de sus beneficiarios, los sistemas de pensiones de la UE no son enteramente meritorios. Las personas mayores parecen estar principalmente satisfechas con sus pensiones solamente en cuatro Estados miembros.

Los resultados de una segunda pregunta a los pensionistas del Eurobarómetro principal parecen indicar un grado relativamente elevado de aspiraciones frustradas y quizá, incluso, un resentimiento latente por el nivel de pensiones que habían acumulado cuando tenían empleo. Les preguntamos que tuviesen en cuenta las contribuciones realizadas durante sus vidas laborales y que dijeran si la pensión que estaban recibiendo ahora les permitía llevar la vida que ellos querían. 
Solamente uno de cada ocho fueron rotundos en su respuesta, y los más certeros estaban en Dinamarca (34 por 100) y Luxemburgo (39 por 100). Sumando los que dijeron sí claramente y los que dijeron probablemente, tenemos lo que se podría denominar mayorías satisfechas en Bélgica, Dinamarca, Luxemburgo y Holanda. Así, en Dinamarca, Luxemburgo y Holanda parece existir, por parte de los pensionistas, un grado elevado de complacencia con el nivel de las pensiones recibidas en su doble vertiente de ser una fuente de ingresos y un premio bien merecido a toda una vida de trabajo. Por el contrario, los países en los que se expresó con mayor rotundidad un rechazo al nivel de las pensiones recibidas por no poder proporcionar a sus beneficiarios el nivel de vida deseado, después de haber contribuido durante toda su vida, son, en orden descendente, los siguientes: Grecia (66 por 100), Portugal (56 por 100), Italia y España (42 por 100) y el Reino Unido (41 por 100).

En el Eurobarómetro principal consideramos importante intentar averiguar la opinión de la población sobre el nivel apropiado de ingresos que debería proporcionar el Estado a las personas mayores. Aquí nos estábamos refiriendo al nivel mínimo de ingresos garantizados. Los resultados refuerzan de manera importante la conclusión de que, en la Unión Europea, existe un fuerte apoyo a que sea el Estado el que garantice un nivel de vida digno a las personas mayores. Esta es una conclusión de inmensa importancia para los gestores de la política económica.

La «opción Beveridge» carecía prácticamente de apoyos. En Dinamarca y Holanda la equidad aparecía como la alternativa más atractiva, lo que puede tener algo que ver con el hecho de que estos dos países, únicos en la UE, tienen sistemas públicos de pensiones basados en la ciudadanía y no en las contribuciones vía el empleo. Pero fue la "opción Bismarck" de equivalencia con los ingresos la clara favorita en una mayoría de países.

Hemos visto en la sección anterior que el mantenimiento del contrato social intergeneracional —-mediante el cual los impuestos y las contribuciones recaudadas a las personas con empleo financian las pensiones de los jubilados- contaba con un apoyo muy sólido. Pero la discusión previa de la suficiencia o insuficiencia de las pensiones plantea inmediatamente la cuestión de cómo deben financiarse. Puesto que los temas relativos al nivel de las pensiones y su financiamiento están entrelazados, aunque raramente considerados conjuntamente en encuestas como ésta, decidimos unirlos en una sola pregunta.

Los resultados que aparecen en el cuadro 9 revelan una clara diferencia de opinión sobre la relación pensiones/impuestos en los países que por sus niveles de pensiones se sitúan en la mitad superior de la UE y los situados en la mitad inferior. Entre los primeros es más probable encontrar la afirmación de que las pensiones son más o menos adecuadas o demasiado bajas, pero que tendrán que permanecer como están; en los segundos, por el contrario, se tiende más a decir que son demasiado bajas y que deberían aumentar, incluso aunque eso también signifique un aumento de las contribuciones o los impuestos. Destaca, en este sentido, la gran diferencia entre la antigua RFA y la RDA. No deja 


\section{CUADRO 9}

Pensiones e impuestos

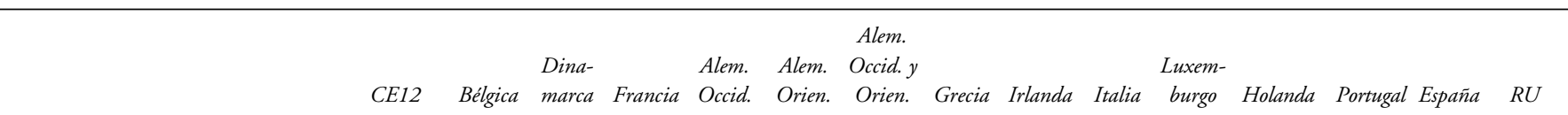

Las pensiones son demasiado bajas y deberían aumentar, aunque también

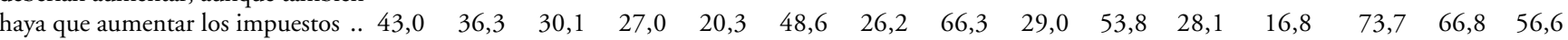

Las pensiones son demasiado bajas, pero no se pueden subir porque no

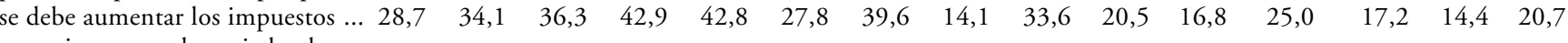
Las pensiones son demasiado altas y

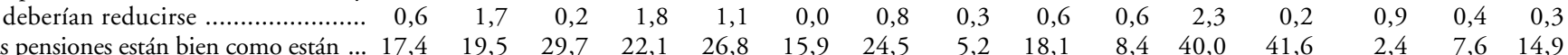

NS/NC

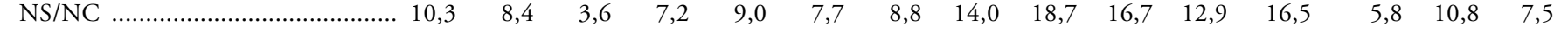


de resultar sorprendente, por otro lado, el muy reducido número de personas, en todos los Estados miembros, que consideran las pensiones demasiado altas.

También resulta notorio que apenas existieran variaciones en las respuestas a esta pregunta entre los diferentes grupos de edad, exceptuando el de 15-24 años, en el que la probabilidad de decir que las pensiones son demasiado bajas y deberían subir, aun cuando se resintieran por ello los impuestos y las contribuciones, era significativamente menor que en los otros grupos.

Relacionado con el tema de la financiación de las pensiones está el de a quién corresponde la responsabilidad de proporcionarlas: ¿al Estado, a los empresarios o a los trabajadores individualmente? En la mayor parte de los países de la UE, una mayoría de la población cree que son las autoridades públicas las que, principalmente, deberían proporcionar las pensiones, financiándolas mediante contribuciones en impuestos. Como promedio, casi la mitad de la muestra de la población general de la UE (el 49 por 100) compartía esa opinión. Los países que se desviaban de esa posición mayoritaria eran Alemania (aunque la antigua RFA estaba a favor y la RDA en contra: 55 y 33 por 100, respectivamente), Holanda y el Reino Unido (aunque sólo un 48 por 100). Las alternativas preferidas de esos tres Estados miembros eran, en el caso de Alemania, pensiones proporcionadas por los empresarios, financiadas principalmente mediante sus contribuciones y las de sus empleados; por otro lado, en Holanda y el Reino Unido, las opiniones estaban divididas entre sus propios sistemas y los contratos privados entre los trabajadores individuales y los fondos de pensiones. No obstante, hay que decir que la proporción a favor de los sistemas privados de pensiones solamente superó el 15 por 100 en Holanda y que, globalmente, este enfoque sólo recibió el apoyo de uno de cada diez entrevistados.

\section{La atención prolongada}

La creciente longevidad es una señal de progreso social y económico que atestigua una intervención fructífera en la muerte y la enfermedad mediante medidas de salud pública. Sin embargo, esto supone que cada vez haya más gente que, probablemente, va a necesitar algún tipo de atención personal o apoyo. Es importante no exagerar este punto: la mayoría de las personas mayores, incluso en su cuarta edad, son capaces de cuidar de sí mismas adecuadamente o sólo con una mínima ayuda de otras personas. Además, si se requiere ayuda, ésta habitualmente proviene de la familia — en la mayoría de los países de la UE el Estado desempeña un papel relativamente secundario en la atención a las personas mayores, ya sea como proveedor o financiador, pero la demanda de dicha atención está aumentando-. Así, un aumento de la proporción de personas mayores que requieran atención tiene implicaciones importantes tanto para las familias (mujeres especialmente) como para los gobiernos. 
¿Quién constituye el principal sostén de las personas mayores que necesitan una atención especial? Si observamos la UE en su conjunto, los hijos adultos son los más mencionados como cuidadores (40 por 100), seguidos de los cónyuges (32 por 100), la asistencia privada remunerada (11 por 100), otros parientes (14 por 100), servicios sociales públicos (13 por 100$)$, amigos (6 por $100)$, vecinos (6 por 100) y organizaciones de voluntarios (3 por 100). Por lo tanto, como ya se había demostrado en investigaciones previas, los miembros de la familia son, con mucha diferencia, los principales proveedores de estos cuidados: dos terceras partes de la atención suministrada a las personas mayores proviene de sus familias, según los datos del Eurobarómetro realizado a personas mayores. Centrándonos primero en los hijos, la mitad de ellos convivía con la persona mayor y la otra mitad ofrecía la ayuda viviendo fuera de su casa. La atención en convivencia es más frecuente en Grecia (39 por 100), Italia (34 por 100) y España (30 por 100), y menos frecuente en Holanda (2 por 100) y Dinamarca (4 por 100). La ayuda de los hijos que viven fuera de la casa es más frecuente en Alemania (32 por 100), Bélgica (27 por 100) y Grecia (26 por 100), y lo es menos en Francia (12 por 100) e Italia (13 por 100).

El papel desempeñado por los cónyuges difiere considerablemente entre los diferentes países de la UE. En Grecia, al 47 por 100 de las personas que reciben cuidados se los proporciona un cónyuge; en Portugal, esa misma proporción es del 44 por 100 y, en Alemania, del 40 por 100. En el otro extremo, tan sólo un 8 por 100 de las personas mayores es atendido por un cónyuge en Holanda, y otro 19 por 100 en Dinamarca.

El papel que desempeña la asistencia privada también difiere ampliamente entre los diferentes Estados. Solamente en tres países llega a superar la quinta parte del total de la atención proporcionada: Francia (27 por 100), Holanda (33 por 100) y el Reino Unido (21 por 100). En contraste, en Grecia, Irlanda, Portugal y España, sólo un 3-5 por 100 de la asistencia tiene este origen.

En cuanto a las prestaciones sociales, no es extraño que aparezcan de manera significativa en los países donde hay un mayor desarrollo de infraestructuras de los servicios públicos. Así, Dinamarca cuenta con el sistema de servicio de atención domiciliaria más extenso de la UE y, en esta encuesta, más de dos tercios del total de personas que estaban recibiendo asistencia eran beneficiarios de servicios sociales. Los siguientes países son Holanda y el Reino Unido, donde sólo algo más de una cuarta parte recibe dichos servicios y, por último, Bélgica y Francia, con algo menos de una quinta parte. En ningún otro país de la UE alcanza esta proporción los dos dígitos (Irlanda fue el más próximo, con un 9 por 100).

En estos resultados se ha observado una cierta influencia de la edad: cuanto más avanzado es el grupo de edad, menor es la probabilidad de que sea el cónyuge quien proporcione la atención y mayor la probabilidad de que se trate de una prestación social. Esta relación de «sustitución» es, en cierta medida, la siguiente: 


\section{CUADRO 10}

Proporción de grupos de diferentes edades que reciben ayuda regular o asistencia de los cónyuges y prestaciones sociales

(En porcentajes)

\begin{tabular}{|c|c|c|c|c|c|}
\hline & $60-64$ & $65-69$ & $70-74$ & $75-79$ & $80+$ \\
\hline 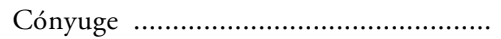 & 54 & 44 & 33 & 25 & 16 \\
\hline 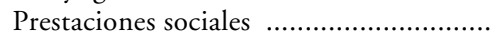 & 8 & 8 & 10 & 13 & 20 \\
\hline
\end{tabular}

El papel desempeñado por los vecinos y el voluntariado también aumenta con la edad de la persona que recibe la atención, pero nunca tan significativamente como el del sector público. Incluso así, está claro que el sector público no compensa totalmente la pérdida de la ayuda prestada por los cónyuges. El papel de la asistencia privada remunerada permanece más o menos constante con la edad, como sucede con el de los hijos y otros familiares. Por lo tanto, estos datos sugieren que existe un espacio sin cubrir, que es el dejado por la pérdida de la ayuda prestada por los cónyuges. Un dato importante es que mientras los cuidados de las personas mayores recaen de manera desproporcionada en las mujeres, hay muchas menos mujeres que hombres recibiendo ayuda de sus cónyuges: solamente el 18 por 100, comparado con el 53 por 100 de los hombres.

A pesar de la existencia de datos como los arriba indicados, que muestran el papel fundamental de la familia en el cuidado de las personas mayores, ha ido perpetuándose el mito de que, en la actualidad, las familias están menos dispuestas a cuidar de sus familiares que en otros tiempos. Este mito ha sido refutado infinidad de veces por investigaciones históricas y contemporáneas. Sin embargo, como muestra el cuadro 11, también en opinión de las propias personas mayores, la familia está menos dispuesta a cuidar de sus mayores. Este resultado es importantísimo no sólo porque contradice lo que parece la realidad objetiva, sino porque también indica la existencia de una preocupante creencia entre las personas mayores. Es cierto que una encuesta más especializada podría haber distinguido entre las «familias en general» y la propia familia de la persona mayor, pero carecíamos de espacio para ello.

Como se puede observar en este cuadro, las opiniones más sólidas a favor de una menor disposición de la familia para atender a sus mayores se expresaron en Francia, Luxemburgo, Italia y España. Por otro lado, los mayores desacuerdos se dieron en Dinamarca, Irlanda y el Reino Unido. No se produjeron apenas variaciones por la edad o el sexo.

No es mera coincidencia que, al haber aumentado el coste de la atención residencial, los gestores de las políticas sociales pertinentes hayan mostrado cada vez más interés en mantener a las personas mayores en sus domicilios —o, aceptando su definición más básica, la atención comunitaria—. Existen 


\section{CUADRO 11}

Las familias, en la actualidad, están menos dispuestas a cuidar a sus mayores que anteriormente (Sólo personas mayores)

\begin{tabular}{|c|c|c|c|c|c|c|c|c|c|c|c|c|c|c|c|}
\hline & CE12 & Bélgica & $\begin{array}{l}\text { Dina- } \\
\text { marca }\end{array}$ & Francia & $\begin{array}{l}\text { Alem. } \\
\text { Occid. }\end{array}$ & $\begin{array}{l}\text { Alem. } \\
\text { Orien. }\end{array}$ & $\begin{array}{l}\text { Alem. } \\
\text { Occid. y } \\
\text { Orien. }\end{array}$ & Grecia & Irlanda & Italia & $\begin{array}{c}\text { Luxem- } \\
\text { burgo }\end{array}$ & Holanda & Portugal & España & $R U$ \\
\hline Muy de acuerdo .......................... & 33,4 & 32,4 & 32,7 & 41,4 & 24,3 & 22,8 & 24,0 & 36,0 & 25,6 & 39,4 & 39,2 & 34,4 & 42,9 & 45,2 & 26,4 \\
\hline Algo de acuerdo ............................. & 34,0 & 37,2 & 26,6 & 32,7 & 39,0 & 30,2 & 37,2 & 35,5 & 26,6 & 34,6 & 24,1 & 27,8 & 36,2 & 34,8 & 31,0 \\
\hline Algo en desacuerdo .......................... & 18,2 & 17,7 & 19,4 & 13,7 & 23,8 & 28,7 & 24,8 & 15,1 & 19,0 & 17,1 & 17,4 & 19,1 & 12,6 & 10,5 & 18,9 \\
\hline Muy en desacuerdo ......................... & 10,4 & 9,2 & 16,4 & 9,5 & 8,9 & 15,4 & 10,2 & 7,8 & 22,5 & 5,1 & 11,9 & 12,5 & 4,9 & 5,5 & 18,3 \\
\hline No sabe ............. & 4,1 & 3,6 & 4,8 & 2,6 & 4,0 & 2,9 & 3,7 & 5,4 & 6,3 & 3,8 & 7,5 & 6,2 & 3,5 & 4,1 & 5,3 \\
\hline
\end{tabular}


otras razones importantes para esta medida, pero ninguna mejor que la expresada preferencia de las propias personas mayores por permanecer en sus casas. Planteamos el tema formulando la siguiente pregunta a la gente: «Algunos dicen que las personas mayores que requieren una atención personal deberían internarse en residencias de tercera edad, mientras que para otros esta ayuda debería consistir en una prestación social que permitiera a las personas mayores permanecer en sus domicilios por tanto tiempo como fuera posible. ¿Cuál de estas dos alternativas se acerca más a lo que usted piensa?»

La gran mayoría de la población (cuatro de cada cinco y nueve de cada diez entre los que expresaron una opinión) pensaba que se debería ayudar a las personas mayores a permanecer en sus casas. Los únicos países en los que más de una quinta parte optaba por las residencias fueron Dinamarca y Portugal (27 por 100). Este dato indica que existe un fuerte apoyo para la opción de la atención comunitaria entre las poblaciones de los Estados miembros. Además, este consenso se extiende prácticamente a todas las edades y es compartido por ambos sexos. La única excepción parcial la constituye el grupo de edad de 15-24 años, en el que una proporción ligeramente mayor (18 por 100) a la de otros grupos apoya la atención residencial, pero desciende en el grupo de 25-34 años al 12 por 100, proporción que permanece hasta el grupo de mayores de 65 años.

Carecemos de espacio, excepto para señalar de pasada que alrededor de uno de cada diez individuos de la muestra de la población mayor de 15 años estaba cuidando a alguien en su propia casa como resultado de una larga enfermedad, una discapacidad o por ancianidad. Además, uno de cada siete proporcionaba ayuda a personas mayores fuera de su domicilio.

La última parte de esta sección se centra en cómo debería financiarse la atención prolongada de un número cada vez mayor de personas delicadas y mayores. En la actualidad, en los países de la UE, esta atención la proporciona el Estado, bien gratuitamente, bien parcialmente subvencionada o, privadamente, siendo financiada por los propios interesados o por el Estado. Alemania ha introducido recientemente un modelo público de seguros para financiar la atención prolongada, y el tema se ha estado debatiendo en diversos países. Pusimos ante la opinión pública una serie de posibles métodos de financiar la atención prolongada y preguntamos que eligieran el método que, en su opinión, fuese mejor.

Los resultados revelaban una oposición sorprendentemente amplia a la utilización del sector privado en este campo. Más de siete de cada diez personas favorecían un modelo público de seguros o unas prestaciones sociales financiadas con impuestos, y, si excluimos a los «no sabe», esta proporción alcanzaba casi a ocho de cada diez personas. Los ciudadanos europeos se han pronunciado con voz clara acerca de este tema: o bien el sector público debería organizar el financiamiento de la atención prolongada o bien debería financiarla y proporcionarla. No había apenas desviaciones de esta posición en términos de la edad o el sexo, aunque el grupo de edad de 55 a 64 años mostraba una predisposición ligeramente mayor a favor de un modelo público de seguros obligatorio. 


\section{LA POLITICA Y EL ENVEJECIMIENTO}

Las encuestas especiales dirigidas a las personas mayores en los diferentes Estados miembros revelan que los ciudadanos de la tercera edad están interesados en temas de actualidad, pero, aunque parecen estar bastante interesados en política, su grado de compromiso con acciones políticas o de grupos de intereses es muy bajo. En el estudio paralelo de las poblaciones generales de estos mismos Estados se preguntaba a los entrevistados por la participación de los mayores en la política y los medios de comunicación.

Con respecto a la vida política, una ligera mayoría de la población general (el 46 por 100 frente al 43 por 100) opinaba que las personas mayores no están desempeñando un papel suficientemente importante. Esta opinión la expresó una mayoría de personas en Bélgica (65 por 100), España (60 por $100)$ y Holanda (56 por 100) y fue, a su vez, rechazada por una mayoría en Grecia (75 por 100), Francia y Holanda (54 por 100$)$ e Italia (50 por 100). Por supuesto que la paradoja que subyace a esta pregunta es que, aunque muchas de las figuras políticas prominentes puedan ser personas mayores, también es cierto, al mismo tiempo, que la gran mayoría de ciudadanos de la tercera edad son menos activos en política, por lo general, que los ciudadanos más jóvenes.

Llevando este mismo tema algo más lejos, preguntamos a continuación si las personas mayores deberían defender más activamente sus propios derechos. Los resultados indican que éste es otro tema sobre el que los europeos están de acuerdo: más del 80 por 100 pensaba que las personas mayores deberían ser más activas en la defensa de sus derechos. No se produjeron apenas desviaciones de ese sentir tan unánime en los diferentes grupos de edad, aunque se observaba una tendencia a que las proporciones que estaban muy de acuerdo fueran aumentando con la edad hasta el grupo de 55 a 64 años, a partir del cual decrecían ligeramente.

Respecto al tema de salir en la televisión y en la radio, una gran mayoría de la población general considera que las personas mayores no tienen una representación suficiente: el 53 frente al 33 por 100 que, por el contrario, piensa que sí la tienen. En Bélgica, Alemania, Grecia, Italia y España, tres de cada cinco personas piensan que las personas mayores no aparecen suficientemente en la televisión ni en la radio.

Para obtener una idea del grado en que los ciudadanos de la UE consideran, o no, adecuadas las prestaciones existentes en sus propios países para las personas mayores y para ver hasta qué punto consideran posibles otras acciones —el relativo "merecimiento» de los mayores-, preguntamos si el Gobierno está haciendo todo lo que debería por este grupo, si hace demasiado o si no hace lo suficiente. Los resultados confirman la alta estima en que se tienen a las personas mayores en Europa. Más de tres cuartas partes de los entrevistados de la UE pensaban que el Gobierno no hace lo suficiente por los mayores, y solamente en cuatro países - Dinamarca, Francia, Luxemburgo y Holanda- 
esta mayoría era inferior a siete de cada diez. Fue en estos mismos cuatro países donde, por el contrario, se dieron las mayores proporciones afirmando que el Gobierno está haciendo todo lo que debería (del 23 por 100 en Dinamarca al 36 por 100 en Holanda). La edad de los entrevistados no influyó apenas las valoraciones de la suficiencia de la acción del Gobierno en la actualidad.

En la misma línea, se preguntó a los entrevistados si consideraban suficiente lo que está haciendo la Comunidad Europea por las personas mayores. Como era previsible, el nivel de incertidumbre aumentó notablemente con respecto a la misma pregunta referida a los gobiernos nacionales. Así, la media de uno de cada tres que respondió «no sabe» lleva sobre sí un importante mensaje acerca del llamado «déficit democrático». Esta incertidumbre alcanzó su punto máximo en Dinamarca (43 por 100), seguido de Holanda y España (35 por 100), y descendió a un 18 por 100 en Portugal. El otro resultado importante que surge de esta pregunta es que una media algo inferior a tres de cada cinco entrevistados opinaba que la UE debería hacer más por las personas mayores. (Si excluyéramos a los «no sabe», casi nueve de cada diez mantenían esa opinión.)

\section{CONCLUSION}

Sería prematuro obtener conclusiones definitivas de lo que sólo es un informe resumido de un gran conjunto de datos; pero los europeos, en buena medida, han perfilado sus propias conclusiones. Dicen muy claramente, por ejemplo, que las personas mayores mantienen un lugar especial como una causa merecedora de acciones gubernamentales y comunitarias en general y, además, quieren más acciones de ambos tipos. Sería equivocado ver esto como una demostración de lástima por las personas mayores. Los resultados revelan una imagen mucho más compleja. Por ejemplo, hay pruebas de una fuerte solidaridad intergeneracional entre jóvenes y viejos — trabajadores y pensionistas- que confiere fuertes dosis de esperanza cara al futuro. Es cierto que en parte se trata de una defensa del interés propio, ya que los jóvenes de hoy pueden esperar hacerse mayores algún día; no obstante, es importante. Existe, entonces, una amplia conciencia de la discriminación por la edad en todos los Estados miembros y la impresión de que se debería hacer algo contra esa injusticia. Por último, hay una fuerte creencia en que las propias personas mayores deberían defender sus derechos más activamente.

Volviendo ahora a las opiniones de los ciudadanos de la tercera edad, existen síntomas de resentimiento por los bajos niveles de las pensiones en algunos países y de ciertos impulsos segregacionistas, pero también hay pruebas de que son parte integrante de sus familias y de que tienen actitudes favorables hacia los jóvenes. Se observan algunas indicaciones inquietantes de inseguridad financiera entre una minoría, pero, en su conjunto, prevalece un sentido de modesta satisfacción con las vidas que llevan, sin muchas quejas ni acción política manifiesta. 
Algo menos de la cuarta parte de las personas mayores estaban muy satisfechas con sus vidas; algo más de la mitad, bastante satisfechas, y solamente una de cada cinco no estaban satisfechas. Las variaciones entre países no pueden obviarse, y el reto al que se enfrenta la Unión Europea consiste en minimizar las derivadas principalmente de las diferencias en las condiciones socioeconómicas. Estas diferencias pueden resumirse en la siguiente espiral estadística: el 68 por 100 de las personas mayores en Dinamarca, el 43 por 100 en Holanda, el 25 por 100 en España, el 6 por 100 en Grecia y el 3 por 100 en Portugal están muy satisfechas con las vidas que llevan. A la inversa, sólo el 3 por 100 de los ancianos daneses no están satisfechos con sus vidas, comparado con el 41 por 100 de los portugueses y el 59 por 100 de los griegos.

Cuánto va a durar la relativa conformidad política de las personas mayores ante semejantes divergencias es un tema para la especulación, pero en las encuestas había indicaciones de un interés latente en las acciones políticas, una clara impresión de compromiso activo con los asuntos de actualidad y un fuerte sentimiento entre la población general de que las personas mayores deberían defender más activamente sus derechos.

\section{(Traducción de Natalia GARCIA-PARDO.)}

\section{RESUMEN}

En este artículo se realiza un análisis comparativo de las actitudes ante el envejecimiento y las personas mayores en los doce Estados miembros de la Unión Europea. Está basado en datos originales de encuesta y se centra en la relación entre las personas mayores y los jóvenes, la jubilación y las pensiones, los trabajadores de edad avanzada y el empleo, la atención social a las personas mayores y la política de la vejez. Por lo tanto, el artículo intenta proporcionar un resumen de las principales dimensiones de las actitudes públicas ante el envejecimiento en la Unión Europea.

\section{ABSTRACT}

This article will examine comparatively attitudes towards ageing and older people in the twelve member states of the European Union. It draws on original survey data and focuses on the relationship between older and younger people, retirement and pensions, older workers and employment, the social care of older people and the politics of old age. Thus the article aims to provide a summary of the main dimensions of public attitudes towards ageing in the European Union. 